\title{
Comparing Interpolations in Frequency Domain and Improvement of Interpolated Images by Using Cube of Pixel Difference
}

\author{
Naoki ONO*, Kiichi URAHAMA \\ Faculty of Design, Kyushu University, \\ Shiobaru 4-9-1, Minami-ku, Fukuoka 815-8540 Japan \\ *Corresponding Author: ono@design.kyushu-u.ac.jp
}

\begin{abstract}
We propose a method for improving image resolution. The method consists of an interpolation and a sharpening process. We research about some interpolations for producing high resolution images and show the properties of the interpolations in the frequency domain. Based on the research, we selected Lanczos function for the interpolation and applied the sharpening method which uses weighted sum of cubic operations of the pixel differences to the interpolated image. Experimental results also show that the sharpening with Lanczos interpolation gives clear high resolution images.
\end{abstract}

Keywords: Interpolation, High resolution, Lanczos function, Cubic operation.

\section{Introduction}

Improving image resolution is a process for making a high resolution image from a low resolution image. For improving image resolution, some interpolation has to be applied and qualities of resultant images are dependent on the interpolation.

In order to produce a high resolution image which has extra-high frequency components, a method using cube of high pass filter has been proposed ${ }^{(1)}$. In the paper, the cube of Laplacian filter is applied to video images to produce high resolution images of them and the validity of the method are shown for producing high frequency components.

For sharpening interpolated images, we proposed a sharpening method which used weighted sum of cubic operations of the differences between a value of pixel and values of the surrounding pixels ${ }^{(2)}$. In the paper, we showed that the method produced a sharp high resolution image which was more similar to a target image than that by any other sharpening method. In the process, the bilinear interpolation was used for enlarging original small size images and the sharpening method was applied the interpolated images.

In this paper, we use another interpolation and apply the sharpening method. Needless to say, since the resultant images are dependent on the interpolation, we research about some interpolation methods used in the field of image processing and show the properties of the interpolation methods in the frequency domain. Based on the research, we select and use an interpolation and then apply the sharpening method to the interpolated images.

Experimental results are also shown that the sharpening with the selected interpolation gives clear high resolution images which preserve the information in the original images adequately.

\section{Properties of Interpolations}

\subsection{Interpolation of Sampled Points and Convolution}

An interpolation of sampled points is accounted to be a convolution with a function for the interpolation. In this section, we describe the properties of some well-known interpolating functions in the frequency domain.

For the convenience, we assume a 1 dimensional sampled data set $\{f(i) ; i=0, \cdots, N-1\}$, such as shown in Fig.1. This data set is assumed to be obtained by sampling an original analog function with sampling distance 1 . According to the sampling theorem, this sampling is equivalent to a filtering by an ideal low pass filter with band width $-1 / 2<\omega<1 / 2$. In other words, the sampling data set has information on $-1 / 2<\omega<1 / 2$ in the frequency domain. 


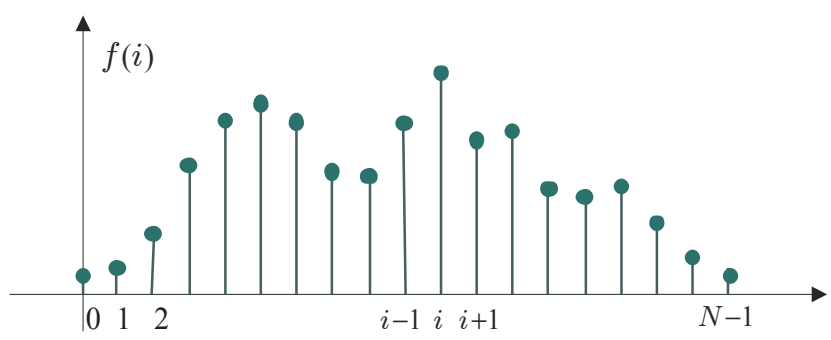

Fig.1. Input sampled data set.

\subsection{The Bilinear Interpolation}

As shown in Figure 2 (a), an interpolated value $f_{l}(x)$ at $x$ between points $i$ and $i+1$ by the bilinear interpolation is calculated by

$$
f_{l}(x)=h_{l}(i-x) f(i)+h_{l}(i+1-x) f(i+1),
$$

where $h_{l}(x)$ is described by

$$
h_{l}(x)=\left\{\begin{array}{ll}
1-|x|, & \text { if }-1 \leq x \leq 1 \\
0, & \text { otherwise }
\end{array} .\right.
$$

The trigonometric function (2) is an interpolating function for the bilinear interpolation and is shown in Fig.2 (b). Figure 2 (a) illustrates a convolution process by eq.(2).

The Fourier transform $H_{l}(\omega)$ of eq. (2) is given by

$$
H_{l}(\omega)=\left(\frac{\sin (\pi \omega)}{\pi \omega}\right)^{2} .
$$

Figure 3 shows power spectrum of $H_{l}(\omega)$, i.e., a transfer function for the bilinear interpolation. For the comparison, the ideal low pass filter concerned with the sampling of original data set is also illustrated in Fig.3.

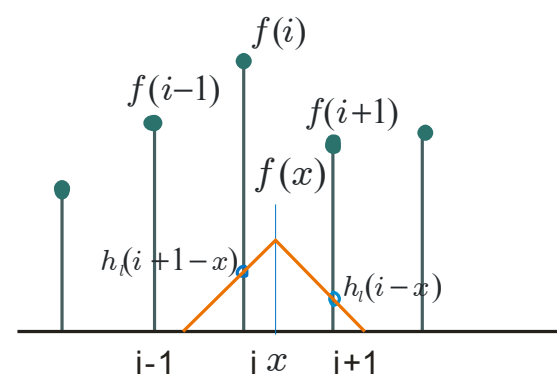

(a) Convolution for the bilinear interpolation.

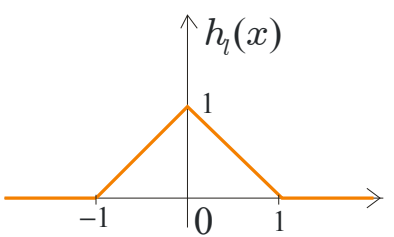

(b) Interpolating function for the bilinear interpolation.

Fig.2. Interpolation by the bilinear interpolation.

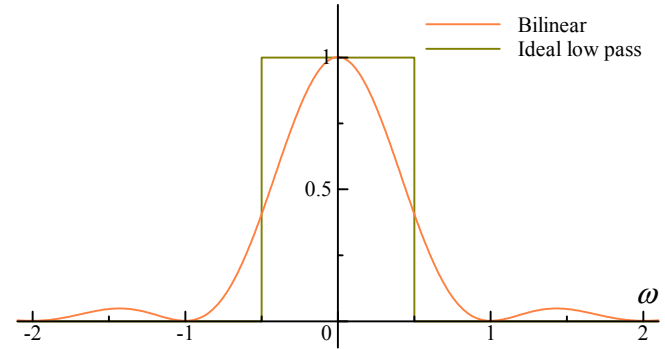

Fig.3. Transfer function for the bilinear interpolation.

\subsection{The Bicubic Interpolation}

As shown in Figure 4 (a), an interpolated value $f_{c}(x)$ at $x$ between points $i$ and $i+1$ by the bicubic interpolation is calculated by

$$
f_{c}(x)=c_{1} f(i-1)+c_{2} f(i)+c_{3} f(i+1)+c_{4} f(i+2),
$$

where weight coefficients $c_{1}, c_{2}, c_{3}, c_{4}$ are calculated by

$$
\begin{aligned}
& c_{1}=h_{c}(1+i-x) \\
& c_{2}=h_{c}(x-i) \\
& c_{3}=h_{c}(i+1-x) \\
& c_{4}=h_{c}(i+2-x)
\end{aligned},
$$

respectively and

$$
h_{c}(x)=\left\{\begin{array}{lll}
|x|^{3}-2|x|^{2}+1, & \text { if } \quad|x| \leq 1 \\
-|x|^{3}+5|x|^{2}-8|x|+4, & \text { if } 1<x \leq 2 . \\
0, & \text { if } 2<|x|
\end{array}\right.
$$

The function (6) is an interpolating function for the bicubic interpolation and is shown in Fig.4 (b). Fig. 4 (a) illustrates eq.(4) and a convolution process by eq.(6).

The power spectrum of Fourier transform $H_{c}(\omega)$ of eq. (6) is shown in Fig. 5 with the ideal low pass filter function.

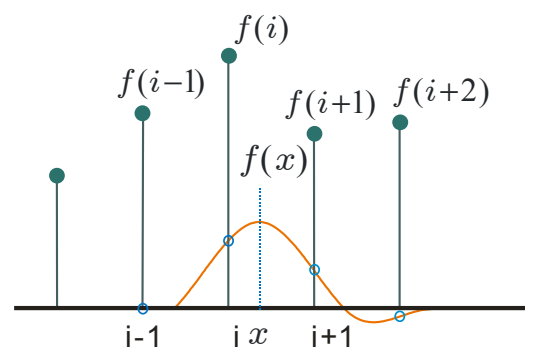

(a) Convolution for the bicubic interpolation.

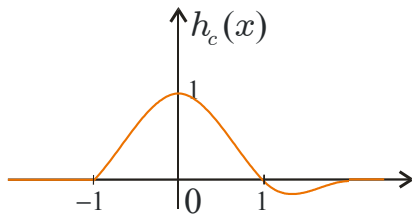

(b) Interpolating function for the bicubic function.

Fig.4. Interpolation by the bicubic interpolation. 


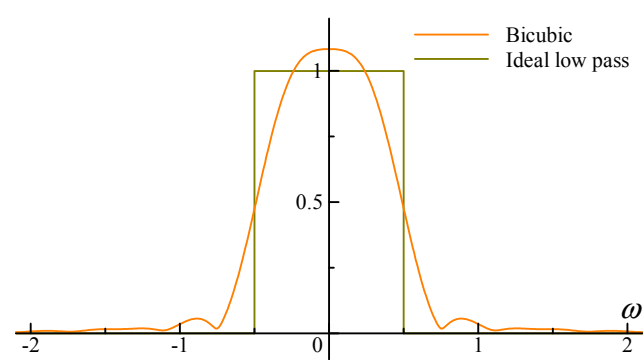

Fig.5. Transfer function for the bicubic interpolation.

\subsection{The Nearest Neighbor Interpolation}

An interpolated value $f_{n}(x)$ at $x$ by the nearest neighbor interpolation is calculated by

$$
\begin{aligned}
& f_{n}(x)=f(i), \\
& i=[x]
\end{aligned},
$$

where [ ] designates to count fractions of 5 and over as a unit and disregard the rest. As shown in Figure 6 (a), this procedure is equivalent to the convolution with a rectangle function

$$
h_{n}(x)=\left\{\begin{array}{ll}
1, & \text { if }-0.5<x \leq 0.5 \\
0, & \text { otherwise }
\end{array} .\right.
$$

The interpolating function $h_{n}(x)$ for the nearest neighbor interpolation is shown in Fig.6 (b).

The Fourier transform $H_{n}(\omega)$ of eq. (8) is Sinc function given by eq. (9)

$$
\operatorname{sinc}(\omega)=\frac{\sin (\pi \omega)}{\pi \omega},
$$

which is shown in Fig.7.

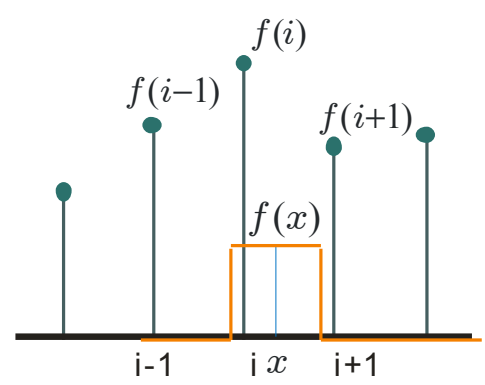

(a) Convolution for the nearest neighbor interpolation.

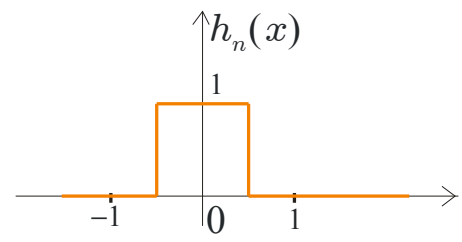

(b) Interpolating function for the nearest neighbor interpolation.

Fig.6. Interpolation by the nearest neighbor interpolation.

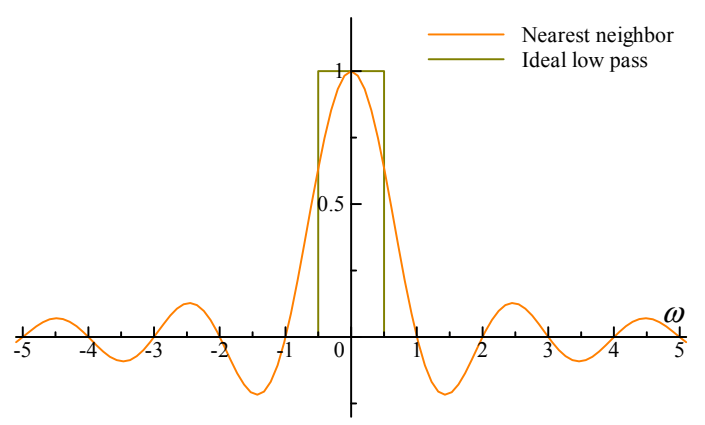

Fig.7. Transfer function for the nearest neighbor interpolation.

\subsection{The Lanczos Interpolation ${ }^{(3)}$}

Lanczos function is often used for image interpolation and generates better results than those by the bilinear interpolation. Lanczos function is an approximation function of Sinc function and described by

$$
L(x)=\left\{\begin{array}{ll}
\frac{\sin (\pi x)}{\pi x} \frac{\sin \left(\frac{\pi x}{n}\right)}{\frac{\pi x}{n}}, & \text { if }|x| \leq n \\
0, & \text { if }|x|>n
\end{array} .\right.
$$

If we set $n=\infty$, eq.(10) is equivalent to Sinc function

$$
\operatorname{sinc}(x)=\frac{\sin (\pi x)}{\pi x} \text {. }
$$

Lanczos functions with $n=2,3,5$ are shown in Fig. 8 and the power spectrum of those functions are shown in Fig.9 respectively. The larger $n$ is, the more similar Lanczos interpolation and the ideal low pass filter are.

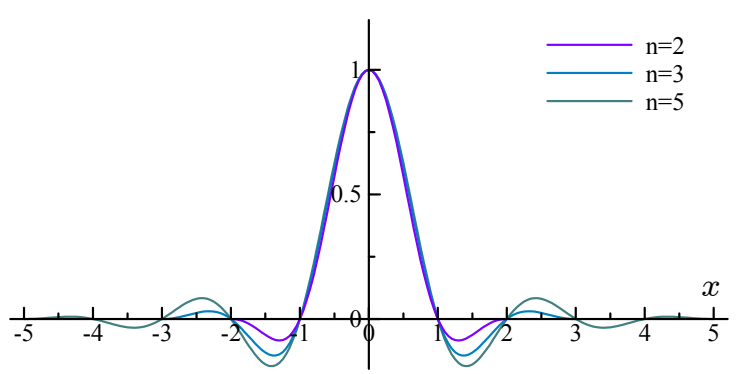

Fig.8 Lanczos functions.

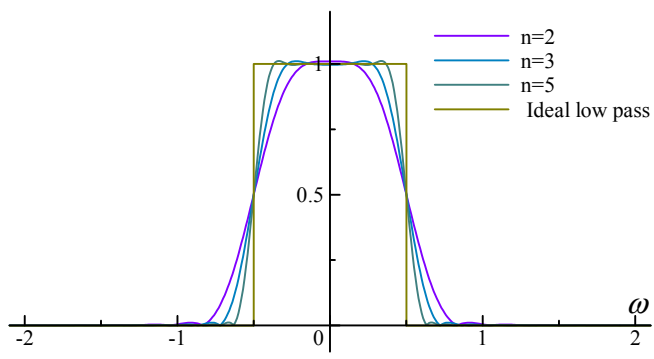

Fig.9. Power spectrums for Lanczos interpolations. 


\subsection{Interpolation by the Ideal Low Pass Filter}

The input sampled data set has spectrum on $-1 / 2<\omega<1 / 2$ in the frequency domain. Expanding spectrum in the frequency domain with preserving the spectrum on $-1 / 2<\omega<1 / 2$ and applying invers Fourier transformation to the expanded spectrum, we have an interpolated values of the input data set. However, the ideal low pass filter yields oscillation which is called Gibbs oscillation $^{(3)}$.

This interpolation is accomplished by a convolution of the input sampled data with eq.(11) in the real domain. This interpolating function for the ideal low pass filter is Sinc function shown in Fig.10. The convolution by a Sinc function tends to yield some oscillations in the result.

\subsection{Comparison of Interpolations}

In order to preserve the information of sampled data set, whole spectrum should be held. The sampled data set has spectrum on $-1 / 2<\omega<1 / 2$ in the frequency domain. Only the interpolation by the ideal low pass filter holds whole spectrum. However in general, the ideal low pass filter yields oscillation around abrupt changes of data.

On the other hand, other interpolations lose some information since the transfer functions of the interpolating functions fall gradually near the boundary $\omega= \pm 1 / 2$ of spectrum in the frequency domain. The attenuation causes degradation of interpolated results.

Since the attenuation of the bilinear interpolating function starts near the origin in the frequency domain, the degradation of result is the largest. The bicubic interpolation has better property in the frequency domain and gives better result than that by the bilinear interpolation. However, a remarkable change around $x=1$ in the bicubic interpolating function tends to yield overshoots in the results.

In Lanczos function, the attenuation in the frequency domain and the oscillation can be controlled by the

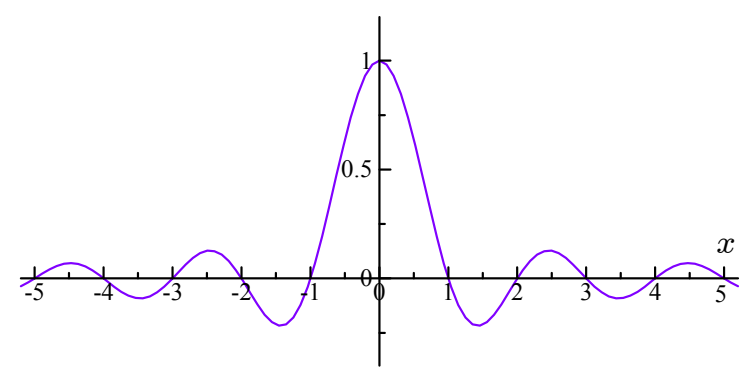

Fig.10. Sinc function for the ideal low pass filter. parameter $n$. Since the oscillation is difficult to remove afterword, we apply an interpolation with suppressing the oscillation and then improve the blur afterword for producing high resolution images. Based on the above research, we select Lanczos function with $n=2$ for the interpolation.

\section{Sharpening for interpolated image}

\subsection{Unsharp masking by liner operation}

The fundamental idea of unsharp masking is to add high frequency components to the input image. In other words, the input image is modified by its own high frequency component.

Given an interpolated image $\left\{d_{i j}(i=1, \cdots, M ; j=1, \cdots, N)\right\}$, the linear unsharp masking (LUM) produces an enhanced image $f_{l i j}$ expressed by

$$
f_{l i j}=d_{i j}+\delta \sum_{l=-p}^{p} \sum_{m=-p}^{p} w_{l m}\left(d_{i j}-d_{i+l, j+m}\right),
$$

where $w_{l m}$ is Gaussian filter

$$
w_{l m}=\frac{e^{-\alpha\left(l^{2}+m^{2}\right)}}{\sum_{l=-p}^{p} \sum_{m=-p}^{p} e^{-\alpha\left(l^{2}+m^{2}\right)}} .
$$

In eq.(12) and eq.(13), $\alpha(>0)$ and $p$ are parameters which control smoothness and size of mask respectively. Furthermore we control the sharpness by a parameter $\delta(>0)$.

\subsection{Sharpening by cube of liner masking}

In the process of unsharp masking, if we adopt the cube of the difference as the high frequency components, slight changes in the image can be enhanced and detail information becomes more visible effectively. According to this idea, enhanced image $f_{c i j}$ is produced by

$$
f_{c i j}=d_{i j}+\delta\left(\sum_{l=-p}^{p} \sum_{m=-p}^{p} w_{l m}\left(d_{i j}-d_{i+l, j+m}\right)\right)^{3} .
$$

This method is, so to speak, the cube of LUM. Using the cube of Laplacian as the high frequency component instead of second term in eq.(14), the sharpening procedure is equivalent to that in the paper ${ }^{(1)}$.

\subsection{Sharpening by using cube of pixel differences ${ }^{(2)}$}

We proposed a sharpening method which was not cube of linear filter but uses weighted sum of cubic operations of the differences between a value of pixel and those of the surrounding pixels, i.e.

$$
f_{d i j}=d_{i j}+\delta \sum_{l=-p}^{p} \sum_{m=-p}^{p} w_{l m}\left(d_{i j}-d_{i+l, j+m}\right)^{3} .
$$


This method also enhanced slight changes effectively. However, since the value of improvement often becomes extremely large, we suppress the value of improvement by setting a limit value $L$. The limitation should be applied to the cube of LUM with eq.(14).

\section{Experiments of Making High Resolution Images}

\subsection{Comparison of Interpolated Images}

An original image, shown in Figure 11, consists of $256 \times 256$ pixels with 256 gray levels. Firstly, we interpolated the original image into enlarged images which consists of $512 \times 512$ pixels by the bilinear interpolation, the ideal low pass filter interpolation, the bicubic interpolation and Lanczos interpolation. Figure 12, Fig.13, Fig.14 and Fig.15 show the interpolated images by the interpolations respectively. In Lanczos interpolation, we set $n=2$ to suppress the oscillation and the processing times.

The image in Fig. 12 by the bilinear interpolation is blurred. Some oscillations near around image frame can be observed in Fig.13 given by the ideal low pass filter. Fig.14 and Fig. 15 look better than Fig.12 and Fig.13.

After interpolating image, it is desired to preserve the frequency components included in an input image before interpolation in order not to change the original image information. On the other hand, the high frequency components which are not contained before interpolation should be created effectively to give a better impression as a high precision image.

Figure 16 shows the FFT result of the original low resolution image. Although the frequency domain of the original image is $256 \times 256$, the components are superimposed in the domain of $512 \times 512$ to compare with the interpolated images. Figure 16 also shows that the original image does not have high frequency components in the outside of $256 \times 256$ low frequency domain. In the graph the values over 0.01 are truncated.

Table 1 shows the mean squared differences of power spectrum between the interpolated images and the original image. In the table, the differences are shown on $256 \times 256$ low frequency domain and other high frequency domain separately. The smaller difference in low frequency domain is, the better interpolation is.

Although an image by the ideal low pass filter interpolation gives no error in this evaluation theoretically, the image may have oscillations. Except for the image by the ideal low pass filter interpolation, an interpolated image by
Lanczos interpolation gives smallest value of the difference than those by other interpolations. In addition, differences in high frequency domain mean high frequency components generated by the interpolations. The high frequency components should be create effectively in order to give a better impression as a high precision image.

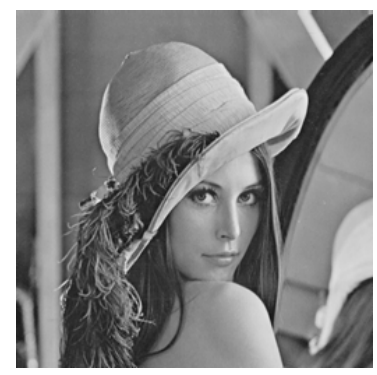

Fig. 11. An original image.

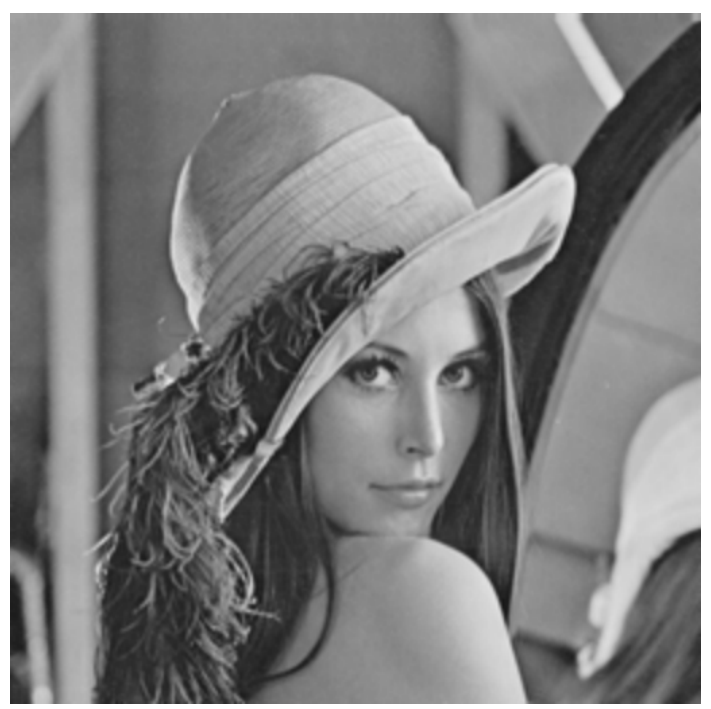

Fig. 12. Result by the bilinear interpolation.

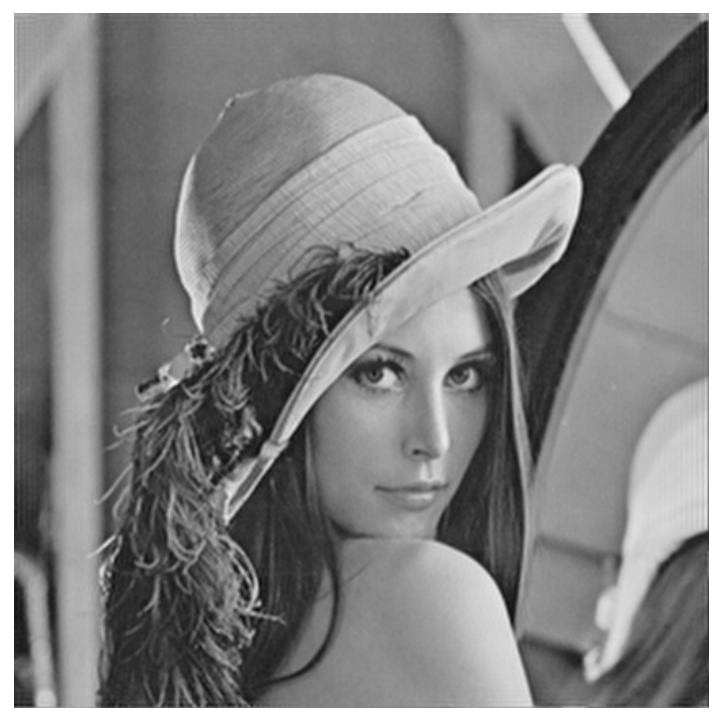

Figure 13. Result by the ideal low pass filter interpolation. 


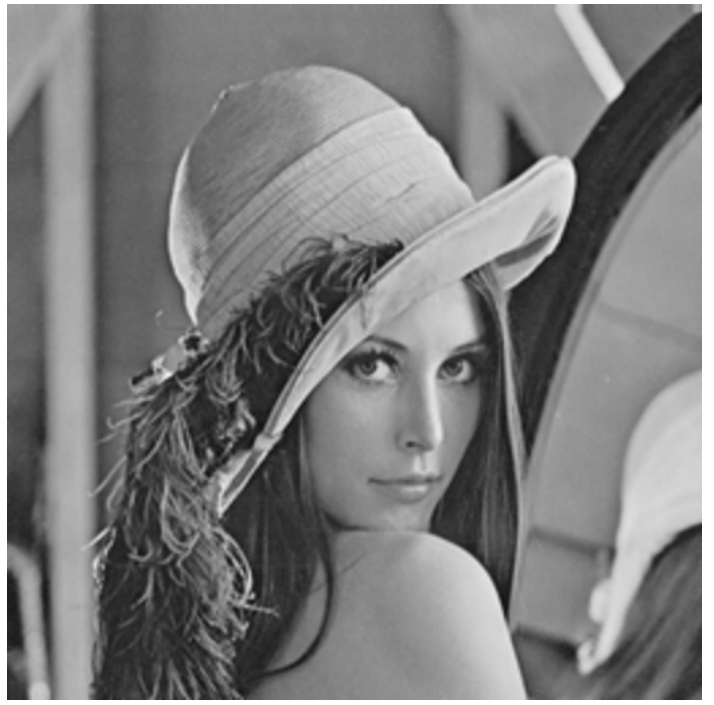

Fig. 14. Result by the bicubic interpolation.

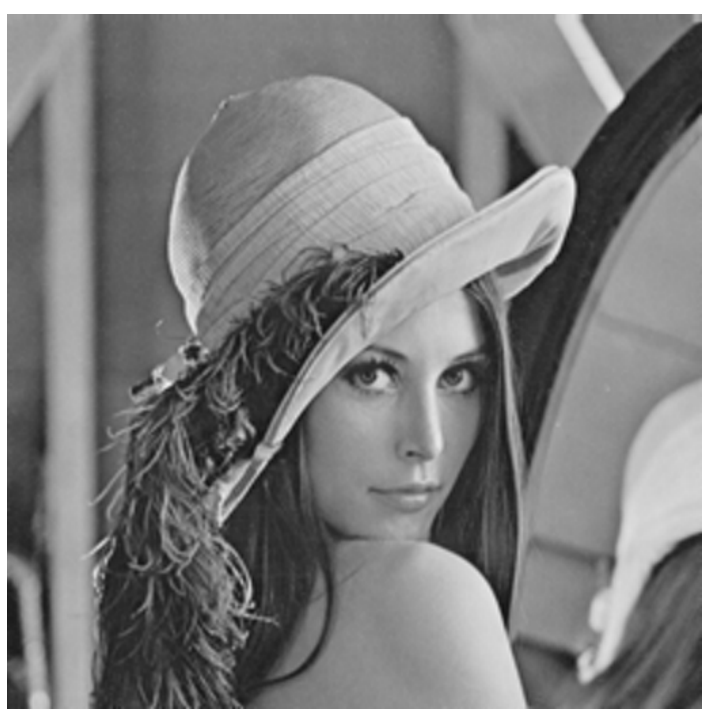

Fig. 15. Result by Lanczos interpolation.

Table 1. The mean squared differences of power spectrum in the interpolated images.

\begin{tabular}{|l|r|c|}
\hline & \multicolumn{1}{|c|}{$\begin{array}{c}\text { Difference } \\
\text { in low } \\
\text { frequency }\end{array}$} & $\begin{array}{c}\text { Generated } \\
\text { spectrum in } \\
\text { high } \\
\text { frequency }\end{array}$ \\
\hline Bilinear interpolation & 0.010439 & 0.002011 \\
\hline $\begin{array}{l}\text { Ideal low pass filter } \\
\text { interpolation }\end{array}$ & 0.000385 & 0.000541 \\
\hline Bicubic interpolation & 0.005247 & 0.002474 \\
\hline Lanczos interpolation & 0.004880 & 0.002425 \\
\hline
\end{tabular}

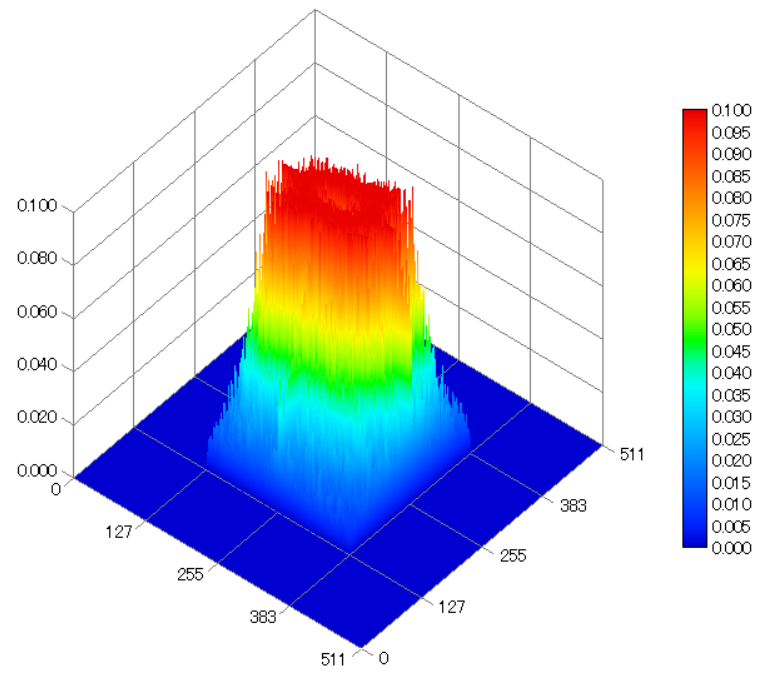

Fig. 16. Power spectrum of the original image.

\subsection{Comparison of Improved Interpolated Images}

We compared the improved interpolated images by Lanczos interpolation and the bilinear interpolation. For improving the interpolated images, we applied the conventional LUM, the cube of LUM and the cube of pixel differences experimentally.

Applying the sharpening methods to the interpolated images by the bilinear interpolation and Lanczos interpolation, we had improved images. Figure 17(a),(b), Fig. 18(a),(b) and Fig. 19(a),(b) show improved images by the LUM, the cube of LUM and the cube of pixel differences respectively. In the experiment, we set the parameters $\alpha=0.3, \delta=2.0$ for Fig.17(a), $\alpha=0.3, \delta=1.0$ for Fig.17(b), $\alpha=0.3, \delta=0.3, L=4 \quad$ for Fig.18(a) and Fig.19(a), $\alpha=0.3, \delta=0.1, L=4 \quad$ for Fig.18(b) and Fig.19(b) respectively.

The images enhanced from Lanczos interpolation are clearer than those from the bilinear interpolation. Although the LUM gave clear texture details, conspicuous edges looks to be enhanced too much with overshoot and undershoot. The cube of LUM with bilinear interpolation (Fig.18(a)) lost the texture details especially in the area of a hat. The image obtained by the cube of pixel difference with Lanczos interpolation (Fig.19(b)) shows clearer details of textures with suppressing excessive edge enhancement such as overshoot and undershoot than those by other improvements.

By improving the resolution of image, the components in higher frequency domain are newly created. We evaluate the components in the low frequency domain included in the original low resolution image and those in the high frequency domain generated by the interpolations respectively. 


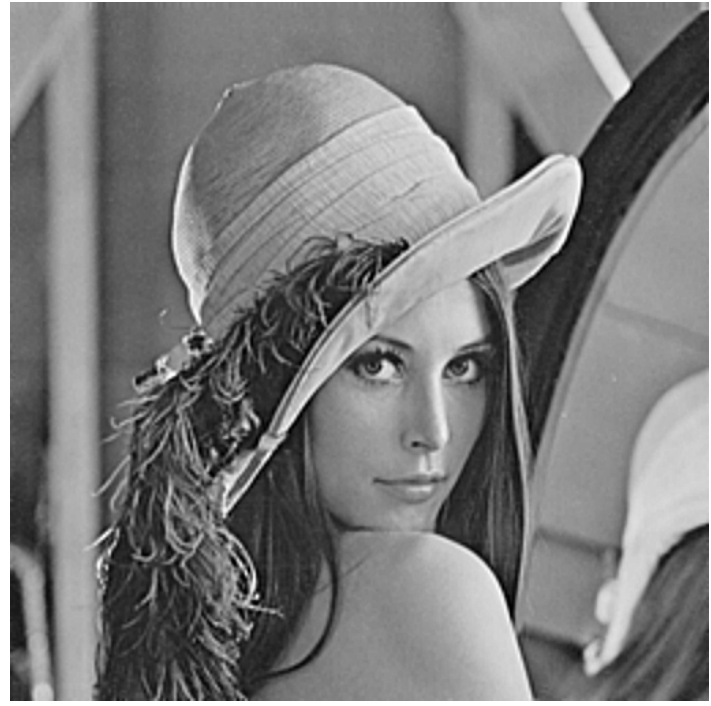

(a). Result from a bilinear interpolated image.

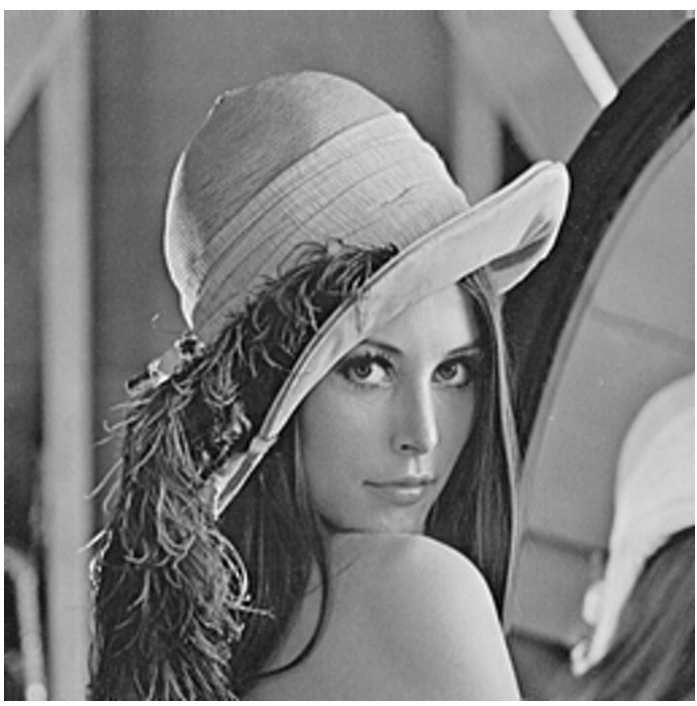

(b). Result from a Lanczos interpolated image.

Fig. 17. Results by applying the LUM.

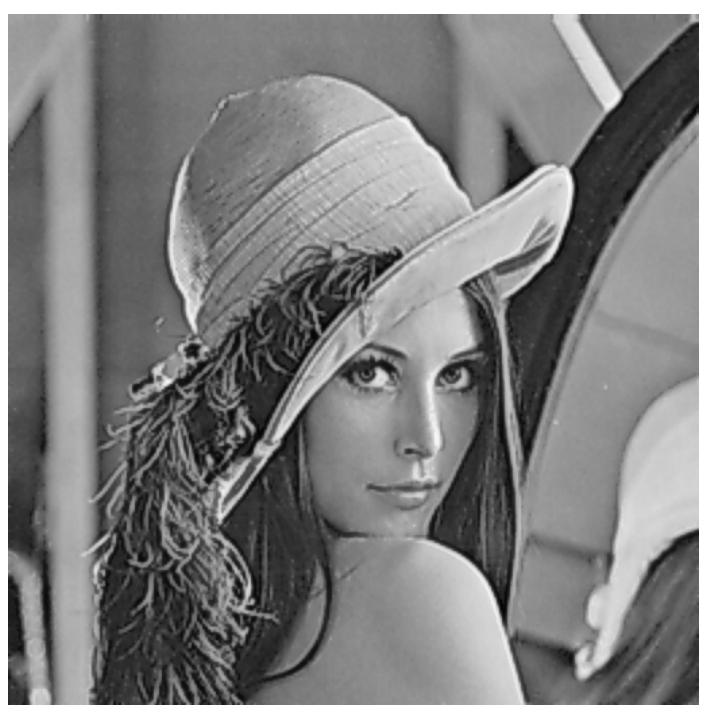

(a). Result from a bilinear interpolated image.

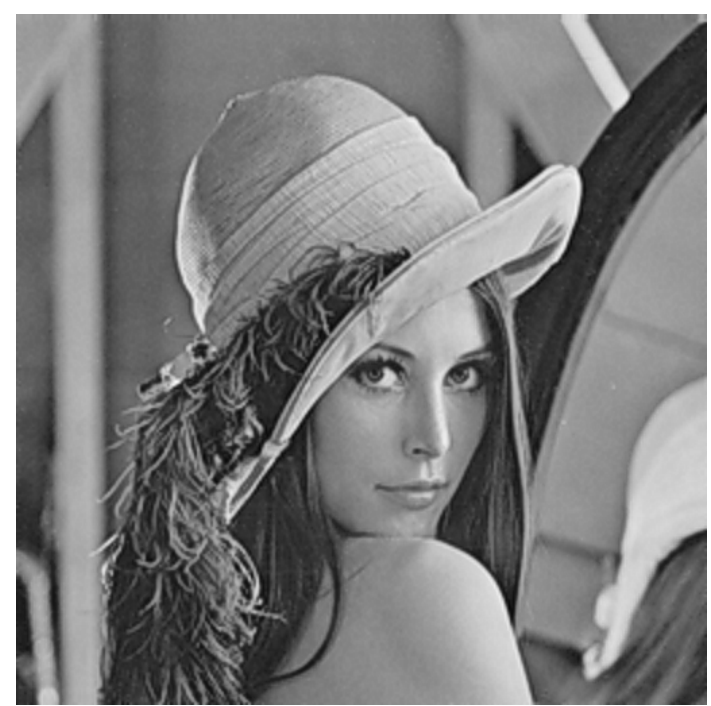

(b). Result from a Lanczos interpolated image.

Fig. 18. Results by applying the cube of LUM.

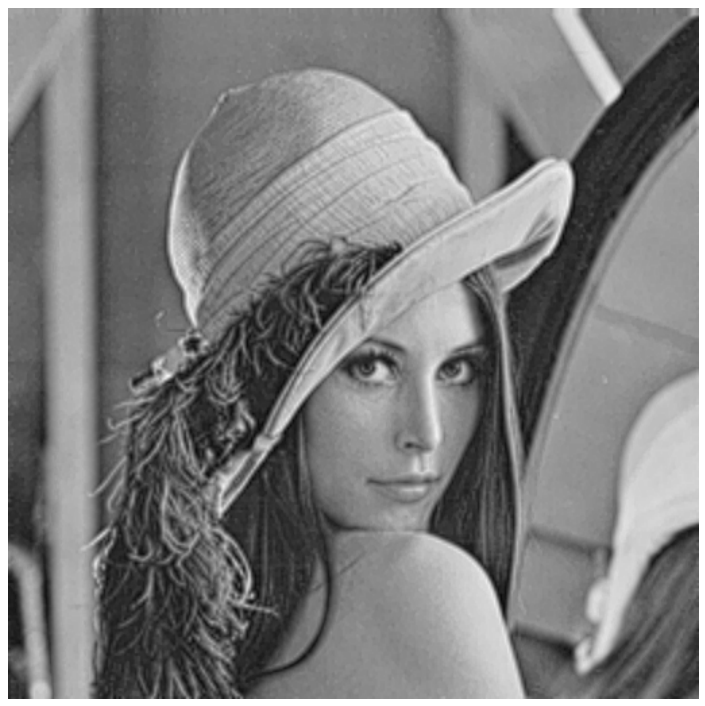

(a). Result from a bilinear interpolated image.

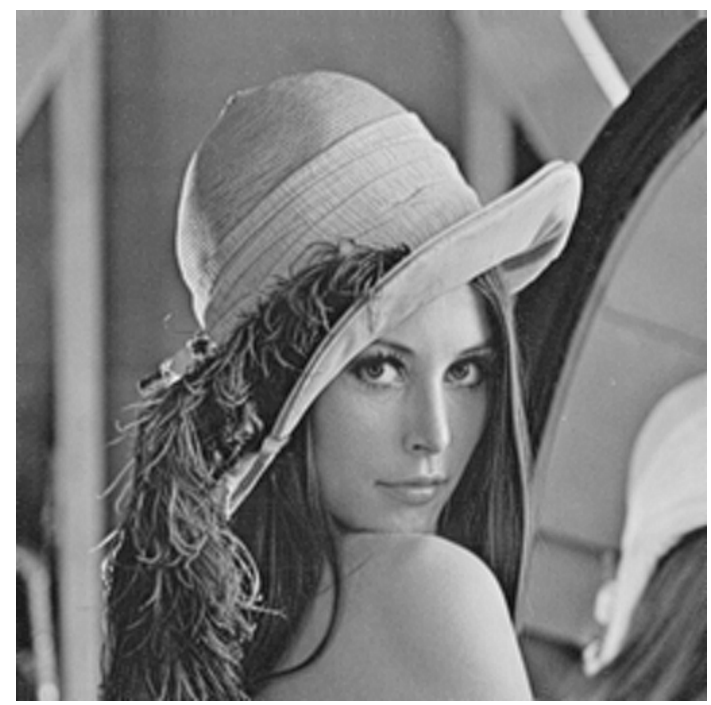

(b). Result from a Lanczos interpolated image.

Fig. 19. Results by applying the cube of pixel differences. 
Table 2 and Table 3 show the mean squared differences of power spectrum between the improved images and the original image Fig.11. For all improved images in table 2 and 3 , the high frequency components increase from those of a bilinear interpolated image and a Lanczos interpolated image in Table 1 respectively. This shows that the sharpening is effective in order to generate high frequency components for interpolated images.

The difference in low frequency domain of a result by the cube of pixel differences is smallest in Table 3. In other words, the sharpening by the cube of pixel differences with Lanczos interpolation is superior in point of keeping information of an original image.

From this experiment, the sharpening by the cube of pixel differences with Lanczos interpolation yields high frequency components with suppressing change of information of original image effectively.

Table 2. The mean squared differences of power spectrum of improved images after the bilinear interpolation.

\begin{tabular}{|l|r|c|}
\hline & \multicolumn{1}{|c|}{$\begin{array}{c}\text { Differences } \\
\text { in low } \\
\text { frequency }\end{array}$} & $\begin{array}{c}\text { Generated } \\
\text { spectrum in } \\
\text { high } \\
\text { frequency }\end{array}$ \\
\hline The LUM & 0.011214 & 0.004463 \\
\hline The cube of LUM & 0.013003 & 0.006188 \\
\hline $\begin{array}{l}\text { The cube of pixel } \\
\text { differences }\end{array}$ & 0.005991 & 0.004132 \\
\hline
\end{tabular}

Table 3. The mean squared differences of power spectrum of improved images after Lanczos interpolation.

\begin{tabular}{|l|r|c|}
\hline & \multicolumn{1}{c|}{$\begin{array}{c}\text { Differences } \\
\text { in low } \\
\text { frequency }\end{array}$} & $\begin{array}{c}\text { Generated } \\
\text { spectrum in } \\
\text { high } \\
\text { frequency }\end{array}$ \\
\hline The LUM & 0.009276 & 0.004090 \\
\hline The cube of LUM & 0.005322 & 0.003885 \\
\hline $\begin{array}{l}\text { The cube of pixel } \\
\text { differences }\end{array}$ & 0.003593 & 0.003270 \\
\hline
\end{tabular}

\section{Conclusions}

In this paper, we proposed a method for improving image resolution. The method consists of an interpolation and a sharpening process.

We researched about some interpolation methods and showed the properties of the interpolations in the frequency domain. Some interpolations yield oscillation or remarkable overshoot. Since the oscillation is difficult to remove afterword, it is better to select an interpolation with suppressing the oscillation and then improve the blur afterword for producing high resolution images. Based on the research, we selected Lanczos function with $n=2$ for the interpolation.

We evaluated the interpolated images in the frequency domain experimentally. An interpolated image by Lanczos interpolation showed better quality than any other interpolations in the experiment.

For the sharpening method, we recommend weighted sum of cubic operations of the differences between a value of pixel and those of the surrounding pixels.

In experiments, we applied the sharpening methods, i.e., the LUM, the cube of LUM and the cube of pixel differences, to interpolated images by the bilinear interpolation and Lanczos interpolation. Interpolated images are improved by the sharpening methods. Especially the sharpening by the cube of pixel differences yields high frequency components with suppressing change of information of the original image reasonably.

Experimental results show that the combination of the sharpening by the cube of pixel differences with Lanczos interpolation gives clear high resolution images effectively.

\section{References}

(1) Ghoshi, "A new signal processing method for video", MMSys '12 Proceedings of the 3rd Multimedia systems Conference, pp. 47-52 (2012).

(2) Naoki Ono and Kiichi Urahama, "Sharpening Interpolated Image by Using Cube of Pixel Difference and Evaluation in Frequency Domain", Journal of the Institute of Industrial Applications Engineers, Vol.4, No.2, pp.56-60 (2016).

(3) C.E.Duchon, "Lanczos Filtering in one and Two Dimensions", Journal of Applied Meteorology, vol.18, pp.1016-1022(1979). 